\title{
Sistema para detecção de estradas e obstáculos baseado em imagens RGB e nuvem de pontos para equipamentos de mineração
}

\author{
Bruno Klafke $* * * *$, Gilberto Resende $*, * *$, Gustavo Freitas***, \\ Andrea G. Campos Bianchi ****, Gustavo Pessin** \\ *Escolade Minas - Universidade Federalde Ouro Preto \\ Ouro Preto,MG, Brasil (e-mail:bruno.klafke@aluno.ufop.edu.br, \\ gilberto.resende@aluno.ufop.edu.br) \\ **Instituto Tecnológico Vale (ITV) \\ Ouro Preto, MG, Brasil (e-mail: gustavo.pessin@itv.org) \\ ***Departamento de Engenharia Elétrica, Universidade Federal de Minas Gerais. \\ Belo Horizonte, MG, Brasil (e-mail: gustavomfreitas@ufmg.br) \\ **** Departamento de Computação-Universidade Federal de Ouro Preto \\ Ouro Preto, MG, Brasil (e-mail: andrea@ufop.edu.br)
}

\begin{abstract}
The mining industry is affected by adverse weather conditions, especially on the rainy season, with heavy fog, which strongly affects operations, reducing and/or interrupting the process of outdoor mining in specific periods of the day. In this sense, the creation of an assisted steering system that helps the operators in these conditions is of great value to improve the usage of mine equipment. This paper presents a proof of concept in which data processing techniques are tested for identification of obstacles and roads in mining environments. The obstacle identification uses an Artificial Neural Networks in RGB images supplied by the camera and the point clustering is done with the density (DBSCAN) method in the point cloud supplied by the laser. Road identification is performed by random sampling (RANSAC) on the point cloud data. Data processing algorithms fuse images and point clouds to create a combined image that helps the identification of the scene in the mining environment. During the tests with data fusion it is possible to identify $95 \%$ of the obstacles, ag ainst the $91 \%$ identification obtained processing individual sens or data.
\end{abstract}

Res umo: A indústria da Mineração s ofre o impacto das condições climáticas adversas, principalmentenos períodos chuvosos, com neblinas intensas, que interferem fortemente nas operações, reduzindo e/ou interrompendo os processos de lavra em determinados períodos do dia. Nesse sentido, a criação de um sis tema de direção assistida que auxilie os operadores nessas condições é de grande valia para melhorar a utilização dos equipamentos de mina. Esse artigo apresenta uma prova de conceito em que são testadas técnicas de processamento de dados obtidos por uma câmera RGB e las er 3D para identificar obs táculos e estradas no ambiente de mineração. Redes Neurais Artificiais (RNA) são empregadas para a identificação de obstáculos nas imagens RGB coletadas por câmeras, e o método de agrupamento de pontos por densidade (DBSCAN) é utilizado para proces sar a nuvem de pontos fornecida pelo laser. A identificação da estrada é realiza por meio do método de consenso de amostragem aleatória (RANSAC) nos dados da nuvem de pontos. Os algoritmos de processamento de dados realizama fusão deima gens RGB e da nu vem de pontos visando criar uma imagem combinada. A fusão dos dados proposta neste artigo permite identificar $95 \%$ dos obstáculos de forma correta, contra 91\% quando considerado processamento sobre os dados fornecidos pelos sensores individuais.

Keywords: RGB camera, laser, cloud points, datafusion, obstacle identification.

Palavras-chaves: Câmera RGB, laser, nuvemde pontos, fusão de dados, identificação de obstáculo.

\section{INTRODUÇÃO}

As minas da Vale no Brasil em que predominam o clima tropical sofrem o impacto das condições climáticas adversas, principalmente nos períodos chu vosos, comneblinas intensas. Para os caminhões do setor de mineração, o risco de acidentes é causado principalmente por comportamento humano e condições adversas de operação. Trafegar em uma mina depende de inúmeras variáveis e uma delas é a condição climática associada a baixa luminosidade, que podem prejudicar a visibilidade do operador.

É de grande importância para o setor mineral viabilizar a operação dos equipamentos mesmo em condições adversas de visibilidade. A redução das horas ociosas permite aumentar a produtividade dos ativos e, consequentemente, a produção da mina. 
Nos últimos anos, veículos e equipamentos com tecnologias embarcadas estão cada vez mais inseridos no setor de mineração. O crescimento dessas tecnologias permite direcionar pesquis as como objetivo de aumentar a segurança na operação, oferecer conforto e outras facilidades para o usuário.

Segundo Freitas (2015), os equipamentos inteligentes vêm se tornando cada vez mais atrativos na cadeia de produção mineral, com aplicações de logísticas automatizadas por uma central de operações. Na mineração vêmcrescendo a utilização dessas soluções e equipamentos, visando melhorar o des empenho, condições de s aúdee segurança, buscando assim afas tar os trabalhadores de áreas de risco.

Com o objetivo de melhorar a segurança e produtividade das operações, o Instituto Tecnológico Vale (ITV) em parcenia o SENAI/CIMATEC está desenvolvendo o projeto "Sistema de Direção As sistida para Caminhões". A solução disponibilizará dados referentes à localização, rota, obs táculos e velocidade que serão captados por dispositivos instalados no veículo. Dess a forma, entende-se que o sistema es tará conectado a um conjunto de sensores, coletando e realizando o processamento e fusão dos dados para que em seguida possa exibir informações úteis para o usuário, no caso o motorista do veículo. Na Figura 1 é apres entado o conceito da interface a ser exibida para o usuário.

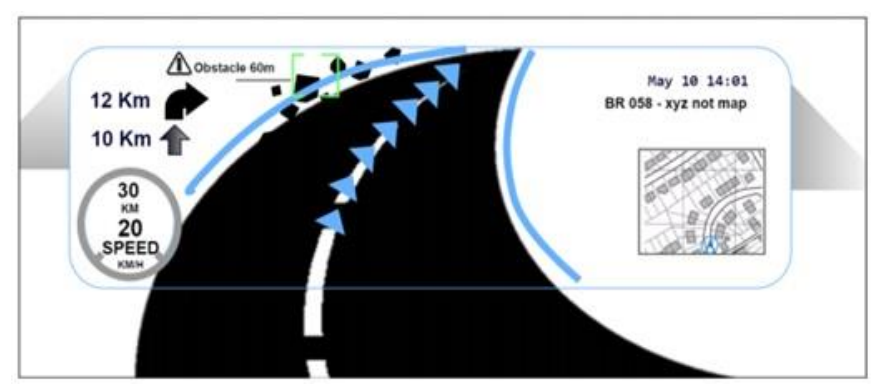

Figura 1. Conceito da interface para o painel do operador (Relatório de Acompanhamento "Projeto Direção Assistida" 2017).

O trabalho apresentado neste artigo consiste em uma etapa preliminar do "Projeto de Direção Assistida", utilizando dados obtidos em boas condições de visibilidade de apenas dois sensores: laser 3D e câmera de vídeo RGB. Também é importante ressaltar que todo o processamento é realizado através de fotos e nuvem de pontos estáticos através de processamento off-line.

Os objetivos deste artigo consis tem em obter dados de cenas do ambiente de mineração a céu aberto, ainda sem condições adversas; validar técnicas de processamento das imagens capturadas pela câmera RGB e a nuvem de pontos do laser como remoção de ruídos, filtrar os dados e evidenciar pontos de interesse; elaborar um algoritmo para identificação de obstáculos e pista de rolagem através dos dados dos sensores operando individualmente; aplicar técnicas de fusão dos dados para identificação das áreas ou pontos de interesse, e aplicar técnicas estruturadas de processamento deimageme nuvemde pontos explorando estratégias já propostas na literatura aplicadas numambiente de mineração.

\section{MÉTODOS PARA PROCESSAMENTODE IMAGENS E NUVEM DE PONTOS}

A imagem digital capturada por câmeras fotográficas ou de vídeo é formada através da radiação proveniente do objeto, podendo ser emis são ou reflexão, tran sformada em um sinal para que possa ser registrado e apresentado de forma adequada à extração de informações (Gonzales et al. 2007). Para Souza (2012), as câmeras são classificadas como sensores passivos, ou seja, não possuem uma fonte de radiação; elas medem a radiação s olar refletida pelos objetos.

O processamento dos dados oriundos do laser permite mapear ambientes e identificar objetos em baixa luminosidade. Este corres ponde a um sens or ativa, ou seja, o dis positivo emite feixes de laser e registra os sinais refletidos, que são menos afetados pelas condições de luminosidade. Portanto, métodos para detecção e extração de objetos emuma nuvemde pontos são de grande importância (Marcon e Ferrão 2016).

Os processos de identificação de estradas e obstáculos através de imagem e nuvemde pontos são realizados separadamente, ou seja, sem interação entre eles. Pos teriormente, são usados os resultados de ambos os sensores para identificação por fusão dos dados. A Figura 2 ilustra o fluxograma para identificação de estradas e obstáculos apresentado neste artigo.

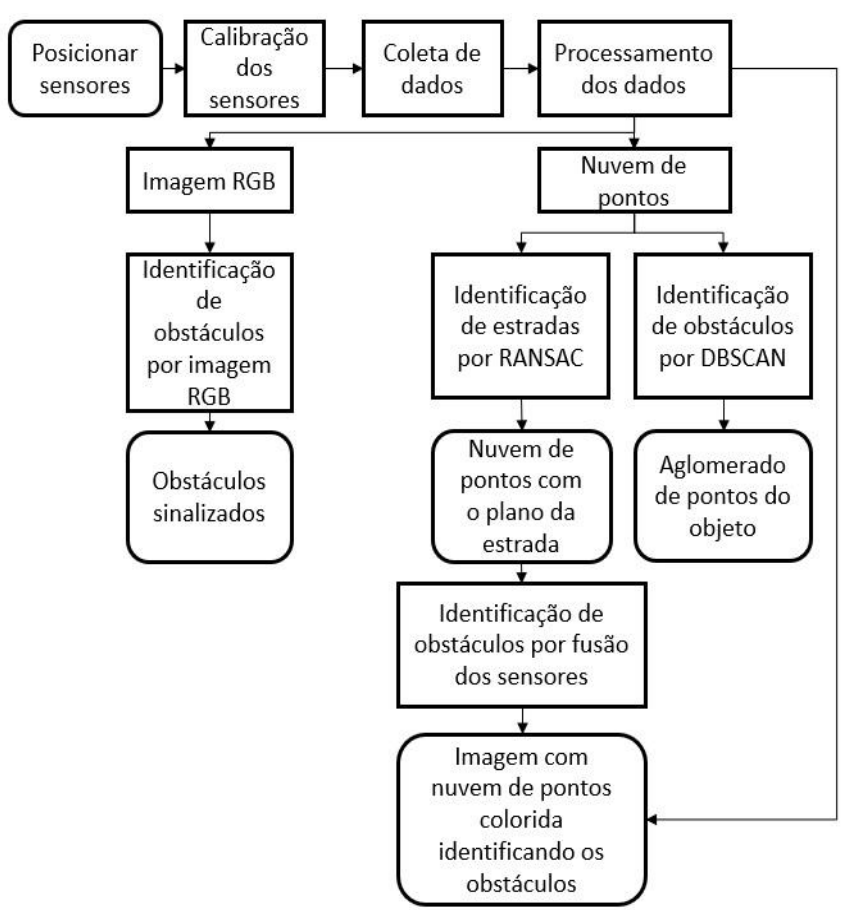

Figura 2: Fluxograma do proces so de detecção de estradas e obstáculos através de imageme nuvemde pontos.

\subsection{Identificação de padrões emimagens RGB através de Redes Neurais Artificiais (RNA)}

As características que mais se destacam nas RNA são a capacidade de aprender através de exemplos (padrões, amostras, etc), a generalização do conhecimento recebido, a atuação como mapeadores de entrada e saída e ser tolerante a falhas (Habermann 2016). 
O Multi-Layer Perceptron (MLP) é umas das RNAs mais conhecidas, descritas como um conjunto de neurônios localizados em camadas organizadas em sequência. Geralmente uma rede neural MLP dispõe de 3 camadas, como ilustrado na Figura 3. A camada de entrada, que recebe os dados a serem ins eridos na rede, a camada escondida, a qual realiza o cálculo dos pesos (conexões) com os dados de entrada, e a camada de saída, que fornece os resultados.

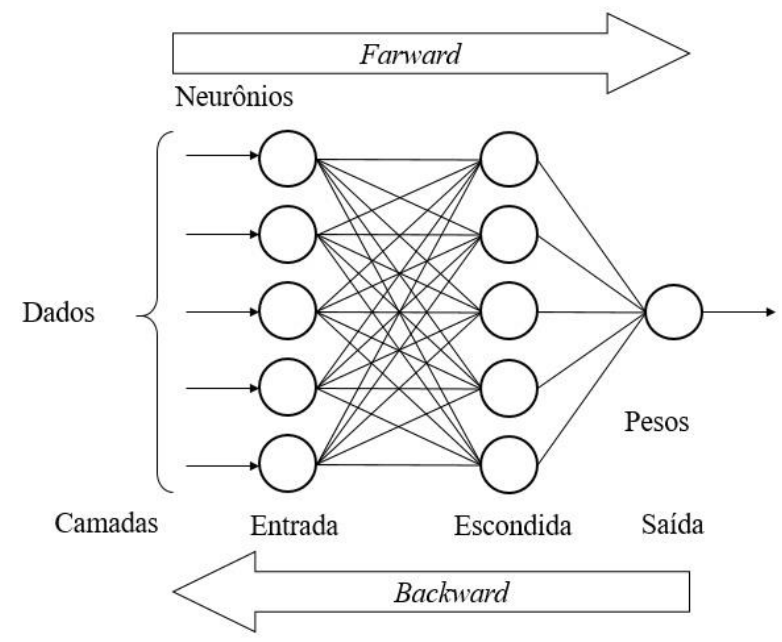

Figura 3. Ilus tração de uma RNA (Habermann 2016).

Cada neurônio de uma camada é conectado com todos os neurônios das camadas posteriores e anteriores, com um determinado peso. Para que os dados de entrada sejam corretamente relacionados às saídas esperadas, é preciso estimar corretamente os pesos que conectam os neurônios da rede. A estimativa é realizada na etapa de aprendizado, que consiste emusar exemplos já classificados e um algoritmo próprio de treinamento (Habermann 2016).

Existem também as Redes Neurais Convolucionais (CNNs), que são baseadas nas RNAs, mas utilizam a convolução em uma ou mais camadas de sua estrutura. As CNNs se mostraram ser eficientes emtarefas de reconhecimento de imagens, porém neces sitam de grande quantidade de dados rotulados para o treinamento (Carneiro e Silva 2017).

As CNNs são compostas por uma sequência de camadas, onde cada camada possui uma função específica na propagação do sinal de entrada. Suas três principais camadas são: convolucionais, de polling e totalmente conectadas. Os atributos dos volumes de entrada são extraídos pelas camadas convolucionais. As camadas de polling tem a finalidade de reduzir a dimensão do volume resultante das camadas convolucionais, e ajudama tornar a representação invariante a pequenas translações de entrada. As camadas totalmente conectadas têma res ponsabilidade de propag aro sinal através da multiplicação ponto a ponto. Para Ren et al. (2017), o resultado de saída de uma $\mathrm{CNN}$ é basicamente a probabilidade de a imagem de entrada pertencer a uma das classes para qual a rede foi treinada.

Neste trabalho, as CNNs são utilizadas para identificação de obstáculos através dos dados da imagem RGB.

\subsection{Processamento da nuvem de pontos.}

Um sistema laser scanner pode ser utilizado para gerar uma nuvem de pontos de três dimen sões. O sistema emite pulsos de laser que são direcionados para o ambiente até ser interrompido por objetos ou pelo alcance do sensor. Tais objetos refletem os pulsos que retornam para o laser. Dessa forma, a dis tância entre o objeto e o sen sor pode ser calculada através do intervalo de tempo existente entre a emissão e reflexão de cada pulso emitido. Es se sistema de funcionamento é conhecido como tempo de voo (Dalmolin e Santos 2004).

Para determinar as dis tâncias dos objetos é medida a variação de tempo $(\Delta t)$ que o sinal precis a para percorrer do sensor até o objeto, e então retornar ao sensor. Com base na velocidade da luz é possível calcular a distância entre o objeto e o sensor, considerando somente metade do valor pois o tempo medido corres pondeao envio e retorno do sinal(Lichti 2002).

A representação obtida pelo scanner é o modelo geométrico conhecido como "nuvem de pontos 3D". Assim, cada ponto $q_{i}$ obtido pelo laser possui 3 componentes com respeito ao sis tema de coordenadas formado pelos vetores orto normais $(X, Y, Z)$, tal que $\overrightarrow{q_{\imath}}=\left[\begin{array}{lll}q_{X} & q_{Y} & q_{Z}\end{array}\right]^{T}$ (Hwang et al. 2016).

Após ser gerada a nuvem de pontos, é neces sário realizar o processamento dos dados para definir conjuntos de pontos pertencentes a nuvemque representamobjetos ou planos.

Para identificação de aglomerados de pontos que representam objetos, o método de agrupamento de pontos Density-

Based Spatial Clustering of Applications with Noise (DBSCAN), descrito emEster(1996), se mostra eficaz Ess a técnica tambémé capazde identificar pon tos isolados que podem ser ruídos de medição e ignorá-los se necessário.

O DBSCAN efetua uma varredura generalizada da nuvem de pontos, identificando distância entre os mesmos, permitindo gerar aglomerados de pontos de qualquer formato. Esse método possui dois parâmetros base: o número mínimo de pontos necessários para criar um aglomerado, denominado MinPts, e a distância base (radial) que delimita a vizinhança de umponto, chamado de Eps. A buscaé iniciada por um ponto qualquer e uma vizinhança é formada quando encontrado uma quantidade mínima de pontos dentro de umdeterminadoraio, iniciando umaglomerado. Érealizada uma varredura por todos os pontos, incluindo cada aponto num aglomerado ou clas sifican do este como ruído caso o ponto não s eja alcançável por outro dentro da circunferência de raio Eps.

Esse processo se repete até que todos os pontos sejam marcados como ruído ou pertencentes a algumconglomerado. A Figura 4 ilustra o método de clas sificação dos pontos por DBSCAN, sendo o ponto roxo o centro da análise, o verde é o limite, o vermelho é ruído e os azuis ainda não foram clas sificados.

Para reconhecimento de planos em nuvem de pontos, uma abordagemamplamente usadaé o Random Sample Consensus (RANSAC). Es se método tem sido aplicado com sucesso à extração de planos que podemrepresentar a estrada (Bazargani 2015). 


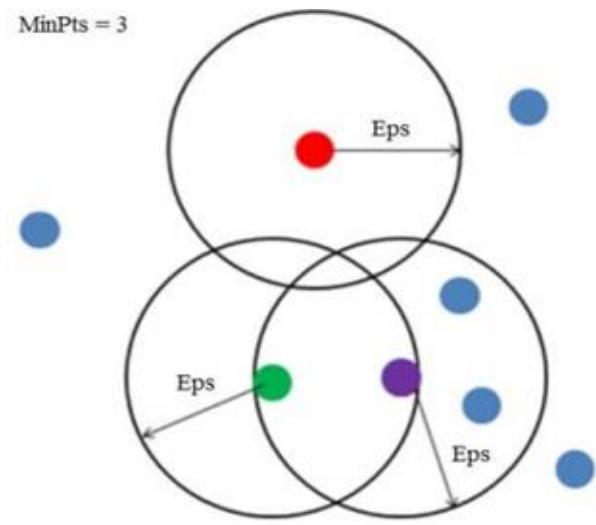

Figura 4. DBSCAN para encontrar agrupamentos (Valêncio, 2013).

A abordagem proposta por Habermann (2016) considera planos definidos na Equação (2):

$$
A X+B Y+C Z+D=0
$$

então, supondo que um dado ponto $\overrightarrow{q_{l}}=\left[\begin{array}{lll}q_{X} & q_{Y} & q_{Z}\end{array}\right]^{T}$ no espaço euclidiano pertence ao plano definido pelo vetor de coeficientes $h=\left[\begin{array}{llll}A & B & C & D\end{array}\right]$ da Equação (2), tem-se em notação vetorial mostradana Equação (3):

$$
\begin{gathered}
{\left[\begin{array}{cccc}
A & B & C & D
\end{array}\right] \cdot\left[\begin{array}{c}
q_{X} \\
q_{Y} \\
q_{Z} \\
1
\end{array}\right]=0} \\
h \cdot\left[\begin{array}{c}
\overrightarrow{q_{l}} \\
1
\end{array}\right]=0 .
\end{gathered}
$$

onde a distância euclidiana $d_{i}$ entre qualquer ponto $\overrightarrow{q_{l}}$ e um plano $h$ é dada por:

$$
d_{i}=\left|h \cdot\left[\begin{array}{c}
q_{l} \\
1
\end{array}\right]\right|
$$

Se o ponto $\overrightarrow{q_{L}}$ pertence ao plano $h$ então $d_{i}=0$, obedecendoà Equação (4). Com a Equação (5), é possível determinar se um determinado ponto $\overrightarrow{q_{l}}$ está dentro ou fora de um limite de distância $t$ do plano $h$. Então, a quantidade depontos danuvem $N=\left\{\overrightarrow{q_{1}}, \overrightarrow{q_{2}}, \ldots, \overrightarrow{q_{n}}\right\}$ que estão dentro do limite de distânciat do plano $h$ pode ser computada conforme Equação (6):

$$
f(h, N)=\sum_{i=1}^{n}\left(\left|h \cdot\left[\begin{array}{c}
\overrightarrow{q_{l}} \\
1
\end{array}\right]\right|<t\right)
$$

Esta é a função de aptidão para o RANSAC quando aplicado à extração de planos em nuvem de pontos. Esta função fomece a quantidade de pontos da nuvem $N$ que se encontram a uma distância menor que $t$ do plano candidato $h$. O limiar $t$ é uma tolerância usada para compensar por irregularidades nos planos e ruídos nos dados.

\section{CALIBRAÇÃODOS SENSORES E FUSÃODOS DADOS}

A fusão dos sensores também é conhecida como sistemas multiparâmetros (Regis 2013). Nas tecnologias que abordamo processamento de dados de modo embarcado, destaca-se a fusão de sensores, que é o processo de combinação dos dados providos de dois ou mais sensores com o objetivo de reduzir as falhas nos processos decisórios. Qualquer interação entre dois sensores, comobjetivo de melhorar umresultadopode ser considerado uma fusão de dados. Para a aplicação proposta, só é pos sível realizar a fusão dos dados da câmera RGB e do laser após a calibração dos sensores.

\subsection{Calibração dos sensores}

Para utilizar os dados dos dois sensores é aplicado ummétodo proposto por Velas et al. (2014), que descreve a calibração automática de imagens RGB com a nuvem de pontos gerada pelo sensor laser. Essa solução consiste em duas etapas: primeiro é realizada uma calibração grosseira, identificando um ponto que possa ser facilmente encontrado na câmera e nos dados do laser, e então são encontrados os demais pontos correspondentes. Este método de calibração basicamente consiste em encontrar os parâmetros de posição e orientação do laser relacionados à câmera RGB, ou seja, o vetor $t$, referente a translação, e a matriz $\mathrm{R}$ que repres enta a rotação, pertencente ao grupo ortogonal es pecial de dimensão $3(R \in$ $S O(3))$ (Corradetti 2016), como ilustrado na Figura 5. Esse problema é conhecido como busca pelos 6 graus de liberdade (6DoF) de trans lação e rotação nos três eixos de coordenadas de um es paço tridimensional. Des sa forma são encontradas as componentes de rotação e translação para a matriz de calibração.
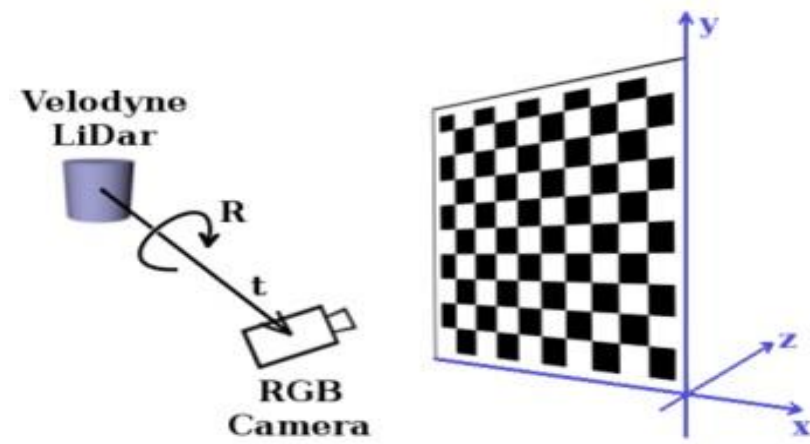

Figura 5. Calibração do laser comcâmera RGB através (Velas et al., 2014).

Inicialmente é usado um marcador que deve ser facilmente identificado na imagem RGB e nos dados do laser. O préprocessamento da câmera inclui a detecção de bordas. A identificação pelo laser é baseada na descontinuidade de posicionamento em um determinado eixo em relação aos pontos vizinhos, conforme a Equação (7):

$$
V_{i}=\max \left(q_{i-1}^{r}-q_{i}^{r}, q_{i}^{r}-q_{i+1}^{r}, 0\right)^{\gamma}
$$

onde o valor de $q_{i}^{r}$ representa o alcance do i-ésimo ponto da nuvem de pontos e $\gamma$ é a constante. O menor $\gamma$ é o valor mais alto atribuído aos pontos de baixa dis torção relativamente aos pontos comalta distorção. Posteriormente, os valores de $V_{i}$ são normalizados entre $(0 ; 1)$.

Os planos são localizados pelo algoritmo RANSAC. Para detecção através da câmera RGB, usa-se o filtro Sobel descrito 
em Dias (2011). É as sumido que o tamanho e as posições do marcador tridimensional são conhecidos, considerado como um objeto plano. O algoritmo verifica então as posições do marcador 3D encontrado pela imagem RGB e pela nuvem de pontos. É necessário transformar as coordenadas homogêneas do ponto 3D da nuvem de pontos para o plano 2D da imagem RGB. A determinação das coordenadas de translação e rotação através da calibraçãoé descrita em Velas (2014).

\subsection{Fusão dos dados}

Após a calibração dos sensores, é possível sobrepor a nuvem de pontos na imagem RGB, facilitando a visualização dos dados em um mesmo resultado. Caso seja alterada a posição dos sensores, então a calibração deve ser realizada novamente.

O principal objetivo da fusão dos dados é identificar a variação de cores RGB entre os pontos da nuvem tridimensional onde o algoritmo de RANSAC identifica uma mudança no plano da estrada, e então é identificado tanto por nuvem de pontos quanto por alteração de cores RGB que existe um obstáculo naquele local. Na Figura 6 é apresentado um flu xograma do código gerado neste estudo para identificar a variação das cores RGB e variação de posição dos pontos vizinhos a um determinado ponto $q_{i}$ clas sificando-o como obstáculo, estrada ou ruído.

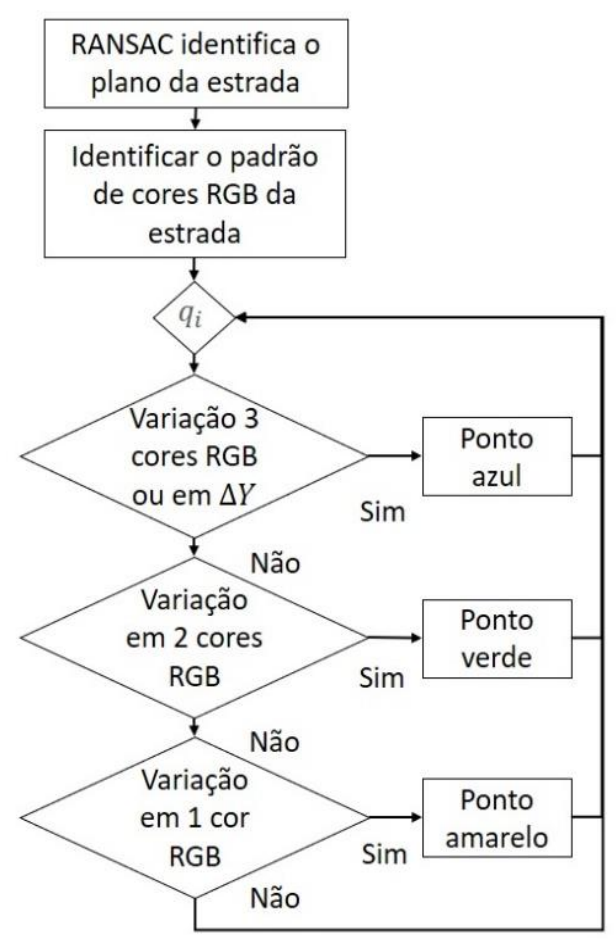

Figura 6. Fluxo para clas sificação de pontos.

\section{TESTES E RESULTADOS}

Inicialmente, para o processo de calibração, os sensores foram fixados na parte superior de uma caixa de madeira e os acessórios na parte interior da mesma para melhor proteção contra intemperes. Após a fixação da câmera e do laser, é necessário calibrar os sensores. Ess a etapa foi realizada no laboratório do ITV utilizando o método de calibração previamente descrito. A matriz de transformação homogênea $G(G \in S E(3))$ definida pela Matriz (8) corresponde à matriz de calibração $M C$ descrita na Equação(9).

$$
G=\left[\begin{array}{cccc}
R^{3 x 3} & & T^{3 x 1} \\
0 & 0 & 0 & 1
\end{array}\right]
$$

onde a submatriz $R^{3 x 3}$ repres enta rotação e $T^{3 x 1}$ é o vetor de trans lação. Dessa forma, a matriz de calibração dos sensores com os componentes $r$ e $t$ referentes a rotação e translação, respectivamente, as sumema seguinte forma:

$$
M C=\left[\begin{array}{cccc}
r_{Z} & r_{X} & 0 & t_{X} \\
r_{Y} & r_{Z} & 0 & t_{Y} \\
0 & 0 & 1 & t_{Z} \\
0 & 0 & 0 & 1
\end{array}\right]
$$

Após a calibração dos sensores, é pos sível realizar a coleta de dados em um ambiente da mineração. A infraestrutura para calibração dos sensores foi montada em um veículo, como ilus trado na Figura 7.

Para tal foramutilizadas uma câmera RGB Axis P1224-E, com campo de visão horizontal de $145^{\circ}$, e um laser LIDAR VLP16 , comcampo de visão de $360^{\circ}$ na horizontale $30^{\circ}$ na vertical usando dezesseis feixes de laser como ilustrado na Figura 8. Outros componentes como bateria móvel, unidade de controle de movimento (Pan Tilt Unit-PTU) e conversores de tensão DC/DC também foram utilizados para o funcionamento independente do conjunto. Todos os itens foram dis ponibilizados pelo ITV.

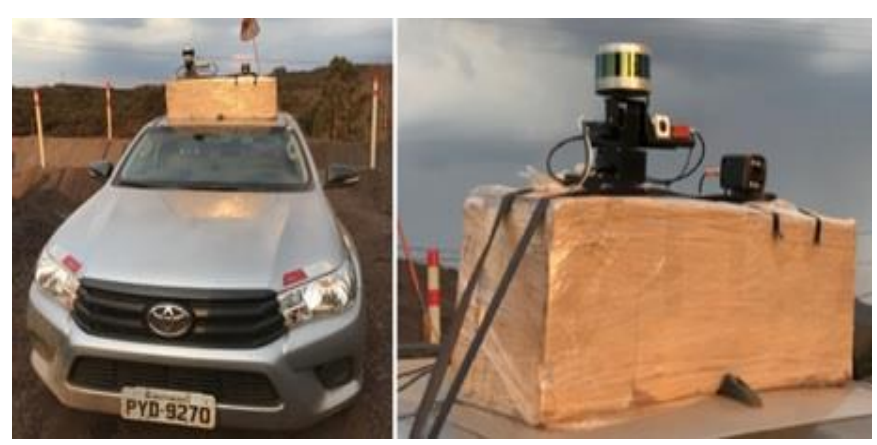

Figura 7. Sens ores fixados no veículo de apoio para coleta de dados.

A coleta de dados foi realizada na Mina de Abóboras, que faz parte do Complexo de Vargem Grande da Vale. Devido a sua localização, condições favoráveis de acesso e vias bem definidas, esta mina foi es colhida para realização das coletas de dados.

Para a execução dos testes foram selecionadas estradas bem definidas e também com veículos como obstáculos. Foram registrados 17 cenários através de nuvem de pontos $3 \mathrm{D}$ e imagens RGB. Além dessa base de dados, também foram utilizadas imagens da internet (https://br.123rf.com) para testes do algoritmo de detecção de obstáculos por RNA. No total, foramutilizadas 60 imagens emcenários comneblina ou 
baixa luminosidade. As imagens da internet foram utilizadas em testes iniciais por possuírem melhor caracterização dos objetos. O processamento dos dados é realizado no software MATLAB.
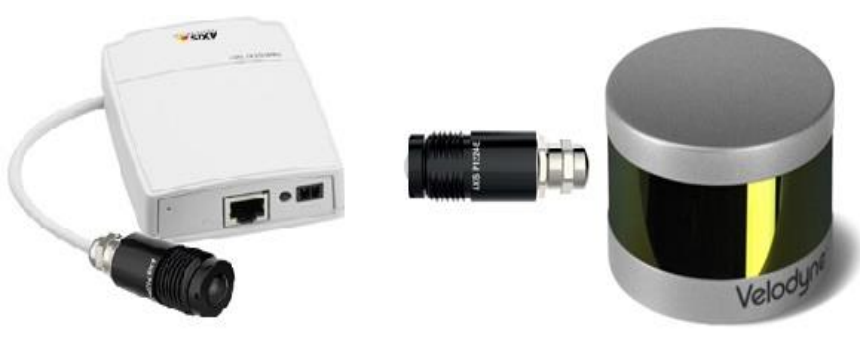

Figura 8. Sensores Câmera RGB AXIS P1224-E e Laser Velodyne LiDAR VLP-16

\subsection{Identificação de Obstáculos em Imagens RGB através de RNAs}

A identificação dos obstáculos na imagem RGB é realizada através da rede neural MatConvnet. É utilizada uma CNN já treinada e testada em diversas configurações. A configuração com 16 camadas foi us ada nessa etapa por apresentar o menor erro nos resultados obtidos emSimonyan e Zis serman (2014). As dimensões do volume resultante da camada convolucional são controladas por três parâmetros: profundidade (depth) com o valor 3 devido as três cores RGB, pas so (stride) como valor de 1 e zero-padding também como valor de 1 para preservar as características principais da imagem.

Antes de usar a CNN com as imagens dos dados coletados em campo, foram utilizadas imagens da internet que continham veículos, pedestres e animais. Es s a etapa te ve como finalidade identificar os obstáculos em imagens obtidas em diferentes condições de visibilidade como: durante o dia, em condições de neblina e durante a noite. A Figura 9 apresenta alguns exemplos de detecção de objetos em imagens com boa visibilidade e comneblina em ambientes deestradas asfaltadas e similares a es tradas dentro de uma mina.

A identificação dos objetos reduz conforme a nitidez dos objetos, conforme apresentado na imagem Tabela 1. Portanto, é de grande importância utilizar sensores com diferentes princípios de funcionando para aumentar a confiabilidade dos resultados.

Tabela 1. Identificação de objetos us ando CNN em diferentes imagens.

\begin{tabular}{|c|c|c|c|}
\hline $\begin{array}{c}\text { Condições de } \\
\text { visibilidade }\end{array}$ & $\begin{array}{c}\text { Quant.de } \\
\text { objetos nas } \\
\text { imagens }\end{array}$ & $\begin{array}{c}\text { Quant.de } \\
\text { objetos } \\
\text { identificados }\end{array}$ & $\begin{array}{c}\text { \% de } \\
\text { identificação }\end{array}$ \\
\hline Dia & 108 & 98 & $91 \%$ \\
\hline Noite & 58 & 49 & $84 \%$ \\
\hline Neblina & 51 & 39 & $76 \%$ \\
\hline
\end{tabular}

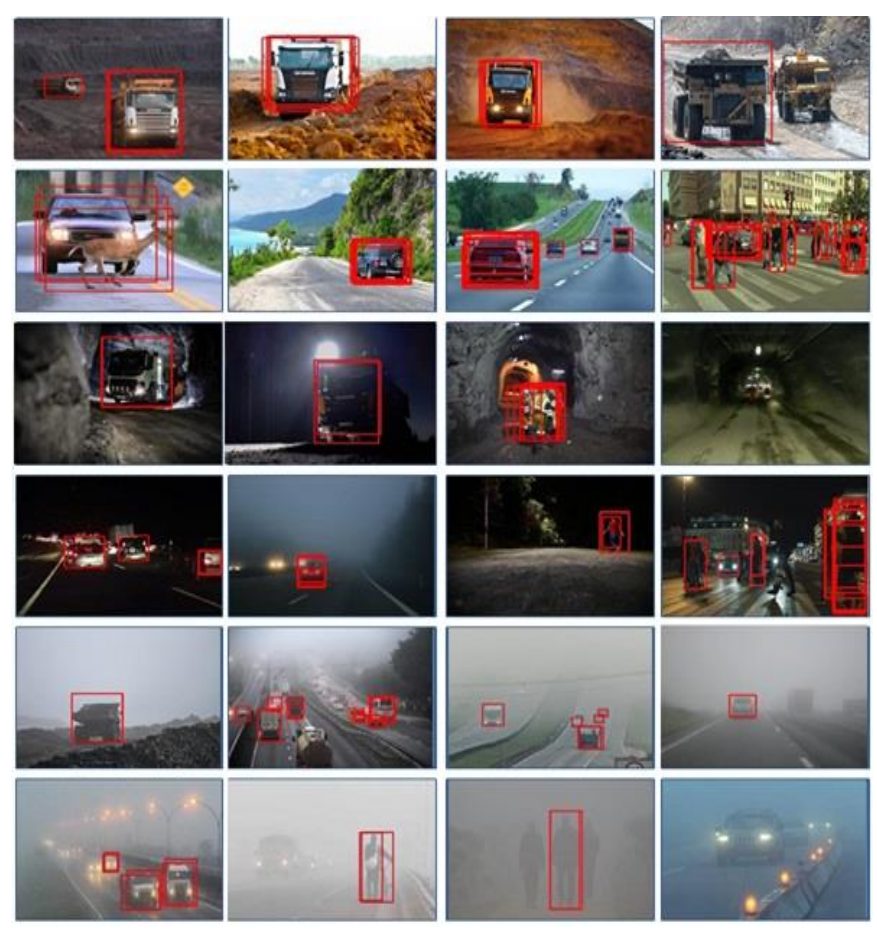

Figura 9. Identificação de objetos emimagens RGB porRNA.

As identificações de objetos nas imagens RGB em ambiente de mineração com boa visibilidade apresentaram $90 \%$ de acertos, conforme apres entado na Tabela 2. Os objetos que se encontram a uma distância superior a $70 \mathrm{~m}$ não foram identificados. A Figura 10 apresenta alguns dos resultados obtidos no ambiente de mineração.

Tabela 2: Identificação de objetos em ambiente de mineração através da $\mathrm{CNN}$.

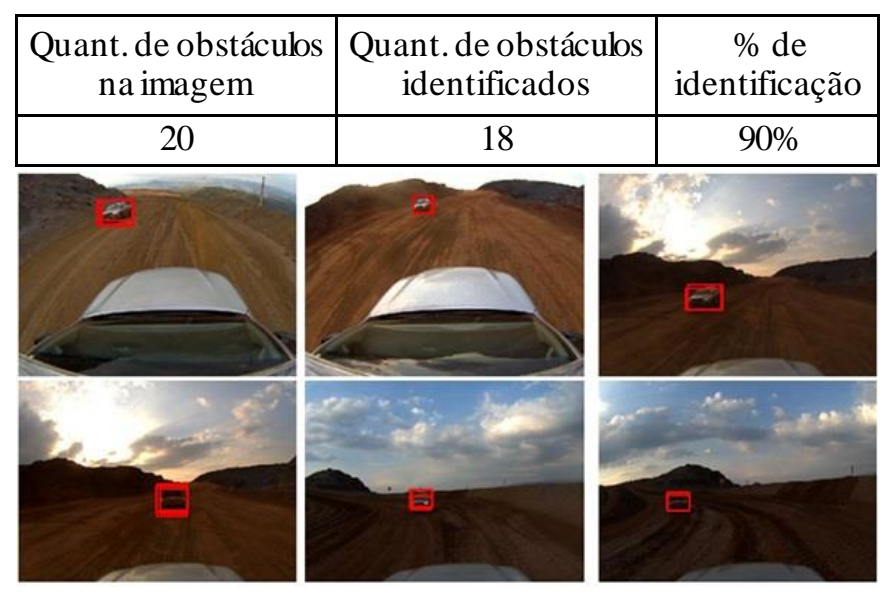

Fig. 10: Identificação de obstáculos emambiente de mineração através de CNN.

\subsection{Identificaçãode Obstáculos e estradas através do processamentoda nuvem de pontos}

Os parâmetros usad os para a configuração doDBSCAN foram de 2 pontos para o MinPts e uma distância radial de $24 \mathrm{~cm}$ para Eps. O número mínimo de pontos foi determinado de acordo com a confiabilidade do laser. Caso sejam encontrados mais 
pontos que o número mínimo adotado, conclui-se a existência de um obstáculo. A distância radialde $24 \mathrm{~cm}$ foi us ada devido à variação no eixo $Z$ entre os feixes de laser do obstáculo mais distante dos cenários coletados. O método de DBSCAN identificou $91 \%$ dos objetos presentes nas nuvens de pontos, conforme apresentado na Tabela 3. Esse método classifica os pontos pertencentes a um obs táculo emvermelho e cons idera os demais como ruído, conforme ilu strado na Figura. 11.

\section{Tabela 3: Identificação de objetos através do método de DBSCAN.}

\begin{tabular}{|c|c|c|}
\hline $\begin{array}{c}\text { Quant. de obstáculos } \\
\text { na nuvemde pontos }\end{array}$ & $\begin{array}{c}\text { Quant.de obstáculos } \\
\text { identificados }\end{array}$ & $\begin{array}{c}\% \text { de } \\
\text { identificação }\end{array}$ \\
\hline 11 & 10 & $91 \%$ \\
\hline & & \\
\hline
\end{tabular}

Figura. 11: Identificação de obstáculos u sando DBSCAN.

Os parâmetros de configuração do método de RANSAC para identificação das estradas dependem de duas variáveis: $t$ é o limiar de dis tância dos pontos do mesmo plano, e $g$ define qual a proporção de pontos que deve ser extraído. Éus ado um valor de $17 \mathrm{~cm}$ para $t$ e 0,4 para g para que sejam encontrados somente os principais planos. Com es ses parâmetros pode-se identificar o plano da estrada mesmo com irregularidades normalmente encontradas em estradas de uma mina. Utilizando o método de RANSAC todas as estradas foram identificadas, conforme Tabela 4. Os planos das estradas possuem grandes dimensões e grande quantidade de pontos, tornando o método eficaz, como apresentado na Figura 12.

Tabela 4: Identificação de es tradas através do método de RANSAC.

\begin{tabular}{|c|c|c|}
\hline $\begin{array}{c}\text { Quant.de es tradas } \\
\text { na nuvemde pontos }\end{array}$ & $\begin{array}{c}\text { Quant.de estradas } \\
\text { identificadas }\end{array}$ & $\begin{array}{c}\% \text { de } \\
\text { identificação }\end{array}$ \\
\hline 15 & 15 & $100 \%$ \\
\hline & \\
\hline
\end{tabular}

Figura 12: Identificação da es trada usando RANSAC.

\subsection{Identificaçãode Obstáculos porfusão dos dados dos sensores.}

A identificação de obstáculos por fusão dos sen sores utiliza as variações de cores RGB e a variação da altura do ponto em relação a nuvemde pontos da estrada para identificar se existe ou não um obstáculo naquele local. São classificados como objetos os pontos que possuem variações acima de $18 \%$ em cada canal RGB, e os pontos que não pertencem ao agrupamento de pontos classificados como estrada pelo RANSAC. O método proposto para fusão de sensores identificou $95 \%$ dos obstáculos, conforme apresentado na Tabela 5. A Figura 13 apresenta a nuvem de pontos coma clas sificação dos obstáculos.

Tabela 5: Identificação de obs táculos através da fusão dos sensores.

\begin{tabular}{|c|c|c|}
\hline $\begin{array}{c}\text { Quant.de obstáculos } \\
\text { nos dados }\end{array}$ & $\begin{array}{c}\text { Quant. de obstáculos } \\
\text { identificados }\end{array}$ & $\begin{array}{c}\% \text { de } \\
\text { identificação }\end{array}$ \\
\hline 20 & 19 & $95 \%$ \\
\hline & & \\
\hline
\end{tabular}

Figura 13: Identificação de obstáculos por classificação dos pontos na imagemRGB.

\section{CONCLUSÕES E TRABALHOS FUTUROS}

O Projeto "Direção Assistida" dis ponibilizará dados referentes à localização, rota, obstáculos e velocidade que serão captados por dis positivos instalados no equipamento.

Este artigo apresenta resultados preliminares utilizando somente dois tipos de sensores, câmera RGB e Laser, para identificação de obstáculos e o posicionamento da pista de rolagemdentro de uma mina a céu aberto.

Considerando os resultados experimentais apresentados conclui-se que é de grande relevância a fusão dos dados oriundos de diferentes sensores, uma vezque umúnico sensor pode apresentar algum tipo de limitação e menor confiabilidade.

As câmeras são amplamente utilizadas para identificações de padrões empregando diversas técnicas de classificação de objetos, incluindo as RNA treinadas com diversas classes. Porém, por se tratar de um sensor passivo, está sujeito a variações de iluminação. O laser possui maior precisão para identificar a distância dos objetos. Por se tratar de um sensor ativo, seu funcionamento não é afetado em ambientes com baixa luminosidade. Porém, é necessário grande quantidade de feixes de las er para mapear de forma representativ a ambientes amplos; quanto maior a distância dos objetos, menor é a densidade da nuvemde pontos, dificultan do a identificação.

A fusão dos dados, onde os dois sensores identificam os obstáculos em conjunto, apresentam resultados mais as sertivos, aumentando a identificação de $91 \%$ para $95 \%$ em relação ao melhor resultado individual dos sensores. 
Como trabalhos futuros é de grande importância realizar coletas de dados em ambiente de mineração em condições climáticas adversas. Dessa forma, as técnicas us adas neste trabalho podems er aperfeiçoadas ou modificadas para melhor percepção do ambiente, realizando medidas mais quantitativas de acertos e erros.

Para o "Projeto de Direção Ass istida" é necessário utilizar vários sensores devidamente calibrados em torno do equipamento para uma percepção em 360 graus do ambiente ao redor. Outra prioridade consiste em realizar a fusão dos dados obtidos comoutros sensores de diferentes princípios de funcionamento incluindo sonares, radares, câmera térmica e GPS. Para a melhor aplicabilidade do sistema em ambiente operacional, é precis o reduzir o tempo de processamento, de forma que a identificação dos objetos e estradas s eja realizada online. Neste sentido, o objetivo final do projeto consiste em embarcar e utilizar o sistema num equipamento de mina.

\section{AGRADECIMENTOS}

Este trabalho foi parcialmente financiado pelo Instituto Tecnológico Vale (ITV); Vale S.A., SENAI/CIMATEC; Empresa Brasileira de Pesquisa e Inovação Industrial (EMBRAPII); Conselho Nacional de Desenvolvimento Científico e Tecnológico (CNPq); Coordenação de Aperfeiçoamento de Pessoal de Nível Superior, Brasil (CAPES), código de financiamento 001; e pela Fundação de Amparo à Pesquisa do Estado de Minas Gerais (FAPEMIG). Os autores gostariamde agradecer à equipe do Laboratónio de Robótica do ITV por apoiar a elaboração do presente trabalho.

\section{REFERÊNCIAS}

Bazargani, M. (2015). Planar surfaces recognition in 3D point cloud using a real-coded multistage genetic alg orithm. pp. 529-540.

Carneiro, A. e Silva, R. (2017). Redes Neurais Convolucionais com Tensorflow: Teoria e Prática. pp. 382-406.

Corradetti, D. e San Martin, L. (2016). Grupos de Lie. Em: Editora UNICAMP pp 89-93.

Dalmolin, Q. e Santos, D. (2004). Sistema Laser scanner. conceitos e princípios de funcionamento. ( $3^{\mathrm{a}}$ edição). pp 67.

Dias, F. et al. (2011). Anais XV Simpósio Brasileiro de Sensoriamento Remoto - SBSR, INPE. pp. 7086-7092.

Freitas, S. (2015). Planejamento estocástico de lavra: metodologias de simulação, otimização e gestão de risco para a mina do futuro.pp. 181.

Gonzalez, R. e Woods R.E. (2007). Digital Image Processing. Addis on-Wesley Pub. Company, 3nd, pp. 89e 976.

Habermann, D., (2016). Localização topológica e identificação de obstáculos por meio de sensor laser 3D (LIDAR) para aplicação em navegação de veículos autônomos terrestres.

Lichti, D., Gordon, S.J. and Stewart, M.P. (2002). Groundbased Laser Scanners: Operation, systems and Applications.pp. 21-33.

Marcon, G. A e Ferrão, V. T. (2016). Proposta de Plataforma de Pesquisa emRobóticaMóvel Autônoma.pp 52.
Regis, A. et al. (2013). Estudo da tecnologia de fusão de sensores em lógica programável aplicado a sistema de monitoramento ambiental. pp.4-7.

Ren, S. et al. (2017). Faster R-CNN: Towards Real-Time Object Detection with Region Proposal Networks. IEEE Transactions on Pattern Analysis and Machine Intelligence, v. 39, n. 6, pp. 1137-1149.

Souza, J. S. D. e Cardoza, J. A. S. (2012). Sensores de Imagem Digitais CCD E CMOS. VII Congresso de Pesquisa e Inovação da Rede Norte e Nordeste de Educação Tecnológica, pp. 1-8.

Valêncio, C. R. et al. (2013). VDBSCAN+: Performance Optimization Based on GPU Parallelism. In: Computing Applications and Technologies (PDCAT). pp. 23-28.

Velas, M. et al. (2014). Calibration of RGB Camera with Velodyne LiDAR. Biosci. Bio-thech Biochem, pp. 5-7. 